\title{
Examining Sex-Differentiated Genetic Effects Across Neuropsychiatric and Behavioral Traits
}

\author{
Joanna Martin, Ekaterina A. Khramtsova, Slavina B. Goleva, Gabriëlla A.M. Blokland, \\ Michela Traglia, Raymond K. Walters, Christopher Hübel, Jonathan R.I. Coleman, \\ Gerome Breen, Anders D. Børglum, Ditte Demontis, Jakob Grove, Thomas Werge, \\ Janita Bralten, Cynthia M. Bulik, Phil H. Lee, Carol A. Mathews, Roseann E. Peterson, \\ Stacey J. Winham, Naomi Wray, Howard J. Edenberg, Wei Guo, Yin Yao, Benjamin M. Neale, \\ Stephen V. Faraone, Tracey L. Petryshen, Lauren A. Weiss, Laramie E. Duncan, \\ Jill M. Goldstein, Jordan W. Smoller, Barbara E. Stranger, and Lea K. Davis, on behalf of the Sex \\ Differences Cross-Disorder Analysis Group of the Psychiatric Genomics Consortium
}

\begin{abstract}
BACKGROUND: The origin of sex differences in prevalence and presentation of neuropsychiatric and behavioral traits is largely unknown. Given established genetic contributions and correlations, we tested for a sex-differentiated genetic architecture within and between traits.

METHODS: Using European ancestry genome-wide association summary statistics for 20 neuropsychiatric and behavioral traits, we tested for sex differences in single nucleotide polymorphism (SNP)-based heritability and genetic correlation $\left(r_{g}<1\right)$. For each trait, we computed per-SNP $z$ scores from sex-stratified regression coefficients and identified genes with sex-differentiated effects using a gene-based approach. We calculated correlation coefficients between $z$ scores to test for shared sex-differentiated effects. Finally, we tested for sex differences in across-trait genetic correlations.

RESULTS: We observed no consistent sex differences in SNP-based heritability. Between-sex, within-trait genetic correlations were high, although $<1$ for educational attainment and risk-taking behavior. We identified 4 genes with significant sex-differentiated effects across 3 traits. Several trait pairs shared sex-differentiated effects. The top genes with sex-differentiated effects were enriched for multiple gene sets, including neuron- and synapserelated sets. Most between-trait genetic correlation estimates were not significantly different between sexes, with exceptions (educational attainment and risk-taking behavior).

CONCLUSIONS: Sex differences in the common autosomal genetic architecture of neuropsychiatric and behavioral phenotypes are small and polygenic and unlikely to fully account for observed sex-differentiated attributes. Larger sample sizes are needed to identify sex-differentiated effects for most traits. For well-powered studies, we identified genes with sex-differentiated effects that were enriched for neuron-related and other biological functions. This work motivates further investigation of genetic and environmental influences on sex differences.
\end{abstract}

https://doi.org/10.1016/j.biopsych.2020.12.024

Despite widespread evidence of sex differences across human complex traits, including neuropsychiatric and behavioral phenotypes (1), the etiology of these differences remains poorly understood. Accumulating evidence suggests that sex differences in complex human phenotypes are likely to include an autosomal genetic component beyond that contributed by sex chromosomes (2-5). Understanding the biological basis of sex differences in human disease, including neuropsychiatric phenotypes, is critical for developing sex-informed diagnostics and therapeutics and realizing the promise of precision medicine (4). Moreover, genetic variants with sex-differentiated effects across multiple traits may influence patterns of comorbidity for neuropsychiatric and related behavioral phenotypes, suggesting the need for cross-disorder genetic analyses to be evaluated in the context of sex-differentiated effects (6-11).

Neuropsychiatric and behavioral phenotypes are generally characterized by a complex and highly polygenic etiology (12). Many of these traits share common genetic risk variants $(13,14)$. Specific genetic loci with pleiotropic effects are known to impact risk for multiple related phenotypes (12). However, it is not yet known whether these pleiotropic effects are consistent across sex.

Several recent studies have investigated sex-differentiated genetic effects for a number of neuropsychiatric traits (15-29). Given evidence of phenotypic sex differences in 
prevalence and presentation as well as genetic correlations between these traits (13), we aimed to systematically test the hypothesis that neuropsychiatric and behavioral phenotypes have a partially sex-differentiated autosomal genetic architecture that may be shared across traits. In this study, we have characterized the 1) sex-dependent genetic architecture for a range of neuropsychiatric and behavioral traits, 2) degree of shared genetic architecture between males and females within each phenotype, and 3) sex-specific patterns of genetic effects shared across traits.

\section{METHODS AND MATERIALS}

\section{Datasets}

We collected sex-stratified genome-wide association study (GWAS) meta-analysis summary statistics for 20 neuropsychiatric and behavioral traits (Table 1; see Sex-Stratified
Datasets in Supplement 1), chosen based on data availability. See Table S1 in Supplement 2 for information about data availability. We used a broad definition of brain-based human complex traits, given the overwhelming evidence of shared genetic effects across such traits (13). We used results from European ancestry GWASs only to minimize any bias that may arise from ancestry differences and because large sexstratified GWAS summary statistics from other ancestries are not currently available. We analyzed autosomal-only common variants with a minor allele frequency $>1 \%$.

\section{Sex-Specific Single Nucleotide Polymorphism- Based Heritability}

For each trait, we calculated sex-specific observed scale single nucleotide polymorphism (SNP)-based heritability $\left(\mathrm{SNP}-h^{2}\right)$ using linkage disequilibrium (LD) score regression (LDSC) with precomputed European ancestry LD scores

Table 1. Summary of Analyzed Datasets of Neuropsychiatric and Behavioral Traits

\begin{tabular}{|c|c|c|c|c|c|c|c|c|c|}
\hline Phenotype (Full Name) & Acronym & $\begin{array}{c}\text { Female } \\
\text { Cases }(n)\end{array}$ & $\begin{array}{c}\text { Female } \\
\text { Controls }(n)\end{array}$ & $\begin{array}{c}\text { Male } \\
\text { Cases }(n)\end{array}$ & $\begin{array}{c}\text { Male } \\
\text { Controls (n) }\end{array}$ & $\begin{array}{c}\text { M:F Case } \\
\text { Ratio }\end{array}$ & Sample Type & $\begin{array}{l}\text { Consortium/ } \\
\text { Group }\end{array}$ & Reference \\
\hline $\begin{array}{l}\text { Attention-Deficit/ } \\
\text { Hyperactivity Disorder }\end{array}$ & ADHD & 4945 & 16,246 & 14,154 & 17,948 & 2.86 & Clinical case-control & PGC+iPSYCH & $(15)$ \\
\hline Alcohol Dependence & ALCD & 2504 & 6033 & 5932 & 9412 & 2.37 & Clinical case-control & PGC & $(16)$ \\
\hline Anxiety Disorders & ANX & 3148 & 191,005 & 1813 & 165,175 & 0.58 & General population (UK) & $\begin{array}{c}\text { Neale } \\
\text { laboratory }\end{array}$ & $(17)$ \\
\hline $\begin{array}{l}\text { Autism Spectrum } \\
\text { Disorder }\end{array}$ & ASD & 7498 & 24,309 & 30,168 & 32,417 & 4.02 & Clinical case-control & $\mathrm{PGC}+\mathrm{iPSYCH}$ & $(18,19)$ \\
\hline Bipolar Disorder & $\mathrm{BD}$ & 10,753 & 14,225 & 7331 & 13,572 & 0.68 & Clinical case-control & PGC2 & (20) \\
\hline Cannabis Use (Ever) & CUE & 17,244 & 71,742 & 17,414 & 50,737 & 1.01 & General population (UK) & $\mathrm{N} / \mathrm{A}$ & $\mathrm{N} / \mathrm{A}$ \\
\hline Insomnia & INS & 19,521 & 39,846 & 12,863 & 40,776 & 0.66 & General population (UK) & $\mathrm{N} / \mathrm{A}$ & $(21)$ \\
\hline $\begin{array}{l}\text { Major Depressive } \\
\text { Disorder }\end{array}$ & MDD & 10,711 & 11,745 & 5021 & 11,226 & 0.47 & $\begin{array}{l}\text { Clinical and population } \\
\text { case-control }\end{array}$ & PGC1 & $(20)$ \\
\hline $\begin{array}{l}\text { Major Depressive } \\
\text { Disorder }\end{array}$ & $\mathrm{N} / \mathrm{A}^{a}$ & 13,492 & 180,661 & 7156 & 159,832 & 0.53 & General population (UK) & $\begin{array}{c}\text { Neale } \\
\text { laboratory }\end{array}$ & $(17)$ \\
\hline $\begin{array}{l}\text { Major Depressive } \\
\text { Disorder Recurrent }\end{array}$ & MDDR & 6026 & 8949 & 2643 & 8162 & 0.44 & Clinical case-control & PGC1 & (20) \\
\hline $\begin{array}{l}\text { Obsessive-Compulsive } \\
\text { Disorder }\end{array}$ & OCD & 1525 & 4307 & 1249 & 2789 & 0.82 & Clinical case-control & PGC & (22) \\
\hline $\begin{array}{l}\text { Posttraumatic Stress } \\
\text { Disorder }\end{array}$ & PTSD & 968 & 2457 & 585 & 4025 & 0.60 & Clinical case-control & PGC & (23) \\
\hline Risk-Taking Behavior & RTB & 32,285 & 143,678 & 51,392 & 100,984 & 1.59 & General population (UK) & $\mathrm{N} / \mathrm{A}$ & $(24)$ \\
\hline Schizophrenia & SCZ & 9837 & 16,763 & 18,346 & 17,122 & 1.86 & Clinical case-control & PGC2 & (20) \\
\hline Smoking (Current) & SMKC & 16,995 & 176,392 & 20,093 & 146,226 & 1.18 & General population (UK) & $\begin{array}{c}\text { Neale } \\
\text { laboratory }\end{array}$ & $(17)$ \\
\hline Smoking (Previous) & SMKP & 62,305 & 131,082 & 65,245 & 101,074 & 1.05 & General population (UK) & $\begin{array}{c}\text { Neale } \\
\text { laboratory }\end{array}$ & $(17)$ \\
\hline & & \multicolumn{2}{|c|}{ Females $(n)$} & \multicolumn{2}{|c|}{ Males $(n)$} & & & & \\
\hline Alcohol Use & ALCC & \multicolumn{2}{|c|}{59,088} & \multicolumn{2}{|c|}{53,088} & 0.90 & General population (UK) & & $(25)$ \\
\hline Alcohol Use & $\mathrm{N} / \mathrm{A}^{a}$ & \multicolumn{2}{|c|}{85,800} & \multicolumn{2}{|c|}{55,120} & 0.64 & General population & & (26) \\
\hline Age at First Birth & AFB & \multicolumn{2}{|c|}{189,656} & \multicolumn{2}{|c|}{48,408} & 0.26 & General population & & $(27)$ \\
\hline Educational Attainment & EA & \multicolumn{2}{|c|}{182,286} & \multicolumn{2}{|c|}{146,631} & 0.80 & General population & & (28) \\
\hline $\begin{array}{l}\text { Number of Children } \\
\text { Ever Born }\end{array}$ & NEB & \multicolumn{2}{|c|}{225,230} & \multicolumn{2}{|c|}{103,909} & 0.46 & General population & & (27) \\
\hline Neuroticism & NEU & \multicolumn{2}{|c|}{144,660} & \multicolumn{2}{|c|}{142,875} & 0.99 & General population (UK) & & (29) \\
\hline
\end{tabular}

F, female; M, male; N/A, not applicable; PGC, Psychiatric Genomics Consortium; UK, United Kingdom.

${ }^{a}$ These summary statistics were not used for analysis (see Sex-Stratified Datasets in Supplement 1 for details). 
(excluding SNPs in the HLA/MHC [human leukocyte antigen/ major histocompatibility complex] region; chr6:25-34M) (30). For 11 binary traits, we also estimated liability scale SNP- $h^{2}$, using sex-specific population prevalence rates from two sources, as described below. For comparison with this primary analysis, we also used a second method, LDAKSumHer (31), to estimate SNP- $h^{2}$, using the LD-adjusted kinships (LDAK) heritability model.

We obtained sex-specific trait prevalence estimates from the United States (32) and cumulative incidence rates from Denmark (33) to compare the SNP- $h^{2}$ estimates using two different sources of information. See Sex-Specific Trait Prevalences for Estimating SNP- $\mathrm{h}^{2}$ in Supplement 1 and Tables S2 and S3 in Supplement 2 for details.

For traits with nonzero SNP- $h^{2}$ estimates (i.e., where confidence intervals did not overlap with zero) in both sexes, we tested whether sex-specific SNP- $h^{2}$ estimates were significantly different by calculating $z$ scores using equation 1 (below) and obtaining corresponding $p$ values from a normal distribution. We corrected for multiple tests using Bonferroni $(n=12$ independent tests for $n=5$ continuous traits and $n=7$ binary traits with nonzero liability scale SNP- $h^{2}$ in both sexes; $p=$ .0042).

$$
z-\text { score }=\frac{S T A T_{\text {female }}-S T A T_{\text {male }}}{\sqrt{S E_{\text {female }}^{2}+S E_{\text {male }}^{2}}}
$$

In equation 1, STAT can be any statistic for which we want to assess the difference between the sexes, including SNP- $h^{2}$, $r_{g}$, and GWAS $\beta$ values; SE is the standard error for the statistic. This test is well calibrated when STAT/SE is normally distributed and the test statistics are independent between sexes and is conservative if the statistics are positively correlated.

\section{Genetic Correlation}

We used LDSC to estimate genetic correlations $\left.\left(r_{g}\right) 1\right)$ between sexes, within each trait, and 2) between each trait pair, within sex (Figure 1A). For between-sex, within-trait correlations, we tested the null hypothesis that $r_{g}<1$ using a 1-tailed test compared with a normal distribution $\left(z=\left(1-r_{g}\right) /\right.$ SE). We applied a Bonferroni multiple-testing correction $(p<$ .0031 based on 16 traits). Next, we tested whether the between-trait $r_{g}$ estimates were different for males $\left(r_{g M}\right)$ and females $\left(r_{g F}\right)$ by using a $z$ score approximation based on block jackknife to estimate the standard error of $r_{g M}-r_{g F}$ in LDSC. As with other LDSC analyses, this approach is robust to sample overlap. We applied a false discovery rate multipletesting correction.

\section{Between-Sex, Within-Trait Genetic Heterogeneity}

Given that only summary statistics from sex-stratified GWASs were available, the analysis of sex-differentiated genetic
A Trait 1
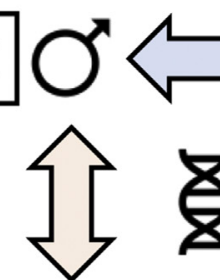

Trait 2

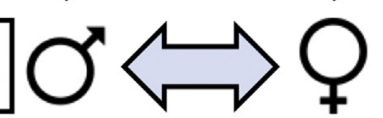

Between-sex, within-trait

- SNP-based $h^{2}$ difference

- Deviation of $r_{g}$ from 1

- SNP heterogeneity

Between-trait, within-sex

- Difference in $r_{g}$ for 2 traits

- Correlation of z-scores across traits
B

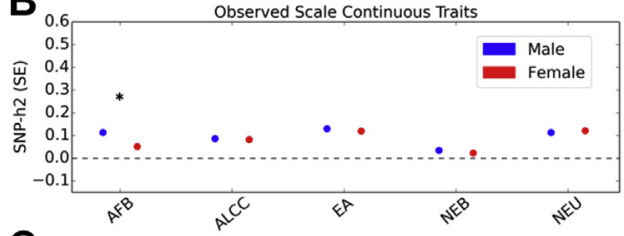

C

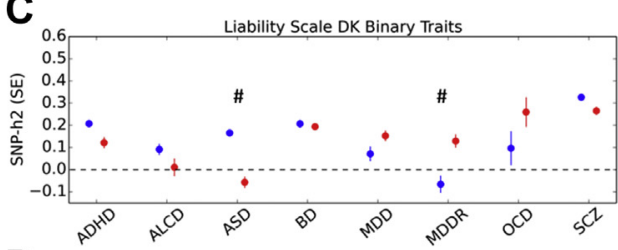

D

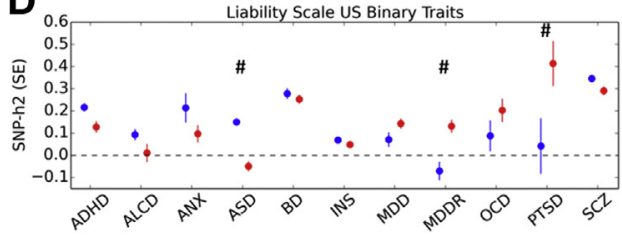

E

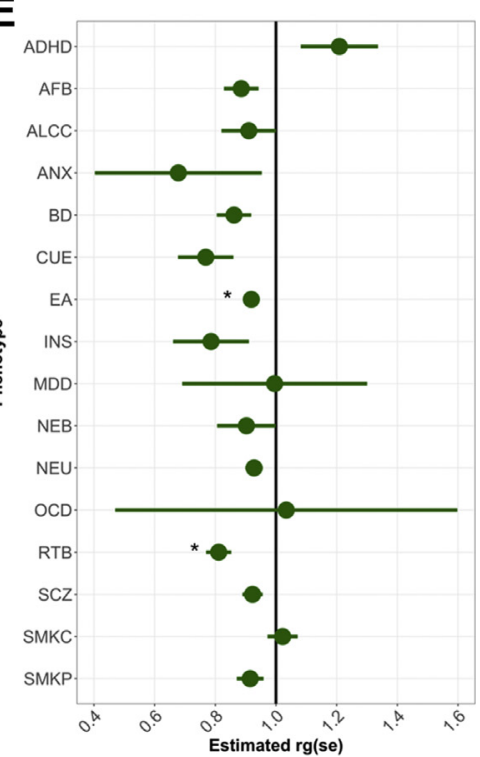

Figure 1. (A) Schematic illustration of the key analyses used to investigate between-sex, within-trait and between-trait, within-sex differences. (B-D) Estimates of sex stratified SNP-based heritability $\left(\mathrm{SNP}-h^{2}\right)$ on (B) the observed scale for continuous traits and the liability scale using population prevalence based on (C) Denmark (DK) and (D) the United States (US). Estimates were obtained from linkage disequilibrium score regression. Points represent the estimated SNP- $h^{2}$ in males (blue) and females (red), while bars represent SE of the SNP- $h^{2}$ estimates. Significant sex difference in heritability is denoted as follows: ${ }^{*} p<.0042$ (adjusted $p$ value threshold corrected for multiple testing using Bonferroni). \#Traits for which significance in difference is not interpretable owing to negative or nonsignificant from zero SNP- $h^{2}$ value for one of the measurements. (E) Within-trait, between-sex genetic correlation $\left(r_{g}\right)$ estimates using linkage disequilibrium score regression. Points represent the estimated $r_{g}$, and bars represent SE of the $r_{g}$ estimates. Significant deviation from 1 is denoted as follows: ${ }^{\star} p<.0031$ (adjusted $p$ value threshold corrected for multiple testing using Bonferroni). ADHD, attention-deficit/hyperactivity disorder; AFB, age at first birth; ALCC, alcohol use; ALCD, alcohol dependence; ANX, anxiety disorders; ASD, autism spectrum disorder; BD, bipolar disorder; CUE, cannabis use (ever); EA, educational attainment; INS, insomnia; MDD, major depressive disorder; MDDR, major depressive disorder recurrent; NEB, number of children ever born; NEU, neuroticism; OCD, obsessive-compulsive disorder; PTSD, posttraumatic stress disorder; RTB, risk-taking behavior; SCZ, schizophrenia; SMKC, smoking (current); SMKP, smoking (previous); SNP, single nucleotide polymorphism. 
effects was limited to the following $z$ score approach. For each SNP in the sex-stratified GWAS of each trait, we assessed between-sex, within-trait heterogeneity using $z$ scores (which are correlated with Cochran's $Q$ statistic but provide directionality of the effect) as in equation 1 . This test quantifies the sex difference in SNP association effect size, similar to, although not the same as, an interaction test (34).

\section{Sharing of Variants With Sex-Differentiated Effects Across Traits}

To assess which traits share sex-differentiated effects (i.e., variants at the extreme ends of the $z$ score distribution), we assessed the Pearson correlation coefficient between $z$ scores (i.e., the differences of $\beta$ values from male-only and femaleonly GWASs) for pairs of traits. Given that there are many nonindependent observations, owing to SNPs in LD, we used a block jackknife approach to estimate the significance of the Pearson correlation $(35,36)$. SNPs were assigned to 1 of 1000 contiguous blocks based on genomic position. For each trait pair, Pearson's correlation was calculated on the full set of $z$ scores and then recalculated after each block was removed, thus estimating the jackknife error and $p$ values.

\section{Gene-Based Analysis, Differential Gene Expression, and Gene-Set Enrichment Analysis of Genes With Sex-Differentiated Effects}

We used the Functional Mapping and Annotation of GWAS (FUMA) SNP2GENE web tool (37) to perform gene-based analysis using MAGMA v1.08 (38,39). We examined whether the genes exhibiting a genome-wide significant sex difference (from MAGMA) demonstrate sex-differentiated gene expression in brain tissues from the Genotype-Tissue Expression project v8 (https://www.gtexportal.org/home/datasets) (20). After mapping SNPs to genes (using a default window size of 0 ), we performed gene set enrichment analysis on the union (across phenotypes) of genes with sex-differentiated effects using GSEA (https://software.broadinstitute.org/gsea/index. jsp). See Gene-Based Analysis, Differential Gene Expression, and Gene-Set Enrichment Analysis of Genes With SexDifferentiated Effects in Supplement 1 for details.

\section{RESULTS}

\section{Sex-Stratified SNP- $h^{2}$ Estimates}

Sex-specific SNP- $h^{2}$ estimates using LDSC are presented in Figure 1B-D, with details provided in Table S4 in Supplement 2. Several traits (posttraumatic stress disorder and recurrent major depressive disorder [MDD] in males and autism spectrum disorder (ASD) and alcohol dependence in females) did not have sufficient power (or had excessive heterogeneity) and we did not detect a polygenic signal, and therefore sex differences could not be assessed. Thus, although we report sex difference estimates for all traits in Table S4 in Supplement 2, these cannot be reliably interpreted for these 4 traits, as one of the sexes exhibited a near-zero SNP- $h^{2}$ estimate. The liability scale SNP- $h^{2}$ estimates using population prevalence from the United States and cumulative incidence from Denmark were highly correlated $\left(r=.97, p=4.7 \times 10^{-10}\right)$ (Figure S1 in Supplement 1). Age at first birth was the only trait with a significant (after multiple testing correction; $p<.0042$ ) sex difference in SNP- $h^{2}$ estimates (females: SNP- $h^{2}=0.052$, SE $=$ 0.004 ; males: SNP- $h^{2}=0.113$, SE $=0.010 ; z$ score $=-5.81$, $p=6.43 \times 10^{-9}$ ).

Observed scale SNP- $h^{2}$ estimates based on LDAK-SumHer were somewhat higher than the estimates obtained in LDSC and moderately correlated with them $\left(r=.69, p=8.5 \times 10^{-7}\right.$ for all traits; $r=.85, p=3.3 \times 10^{-11}$ excluding the 4 traits for which SNP- $h^{2}$ could not be reliably estimated in LDSC); see Table S5 in Supplement 2 and Figures S1 and S2 in Supplement 1 for details. Higher estimates from the LDAK model relative to the LDSC model have been previously observed $(31,38)$. In contrast to LDSC results, age at first birth did not show a significant sex difference $(z$ score $=1.94, p=$ .052), with an effect in the opposite direction to that observed using LDSC. Using LDAK, the liability scale (adjusted based on each population) SNP- $h^{2}$ estimates differed by sex for the following traits: recurrent MDD (United States: $z$ score $=-4.68$, $p=2.84 \times 10^{-6}$; Denmark: $z$ score $\left.=-4.46, p=8.06 \times 10^{-6}\right)$, ASD (United States: $z$ score $=2.94, p=.0033$; Denmark: $z$ score $=3.28, p=.0011$ ), and schizophrenia (Denmark: $z$ score $=-3.16, p=.0016$ ). These results were not observed using LDSC, and indeed SNP- $h^{2}$ could not be estimated reliably in LDSC for ASD in females or recurrent MDD in males. The biggest discrepancies between estimates obtained from LDSC and LDAK were for the traits with the smallest sample sizes (Figure S3 in Supplement 1). The SNP- $h^{2}$ results for attention-deficit/hyperactivity disorder (ADHD) and ASD were similar, albeit somewhat higher, for both LDSC and LDAK when using estimates based on a Danish child-specific study (39) compared with using prevalence estimates from the whole Danish population (Tables S4 and S5 in Supplement 2) (33).

\section{Between-Sex, Within-Trait Genetic Correlation Analysis}

We quantified the genetic correlation between males and females for each trait (excluding the 4 traits where SNP- $h^{2}$ could not be estimated in one of the sexes) (Figure 1E and Table S6 in Supplement 2). We found moderate-to-high genetic correlations for all traits $\left(r_{g}=0.68-1.21\right)$; these all differed significantly from zero, and we also detected a significant difference from 1 for risk-taking behavior $\left(r_{g}=0.81\right.$, SE $\left.=0.04\right)$ and educational attainment $\left(r_{g}=0.92, \mathrm{SE}=0.02\right)$, after correcting for multiple tests $(p<.0031)$, suggesting a modest degree of common variant heterogeneity in males and females for these phenotypes.

\section{Between-Sex, Within-Trait Heterogeneity Across Variants}

To assess sex differences in genetic effects of individual common variants, for each trait we computed $z$ scores and corresponding $p$ values for each SNP, using equation 1 . Figure $S 4$ in Supplement 1 shows the quantile-quantile plots of the $z$ score $p$ values for all traits. While there were no genomewide significant $\left(p<5 \times 10^{-8}\right)$ differences between male and female $\beta$ values for any individual SNP, we observed deviation from the expected null distribution (Figure S4 in Supplement 1) for ADHD, lifetime cannabis use, MDD, number of children born, and schizophrenia. Figure 2A shows a Miami plot for 

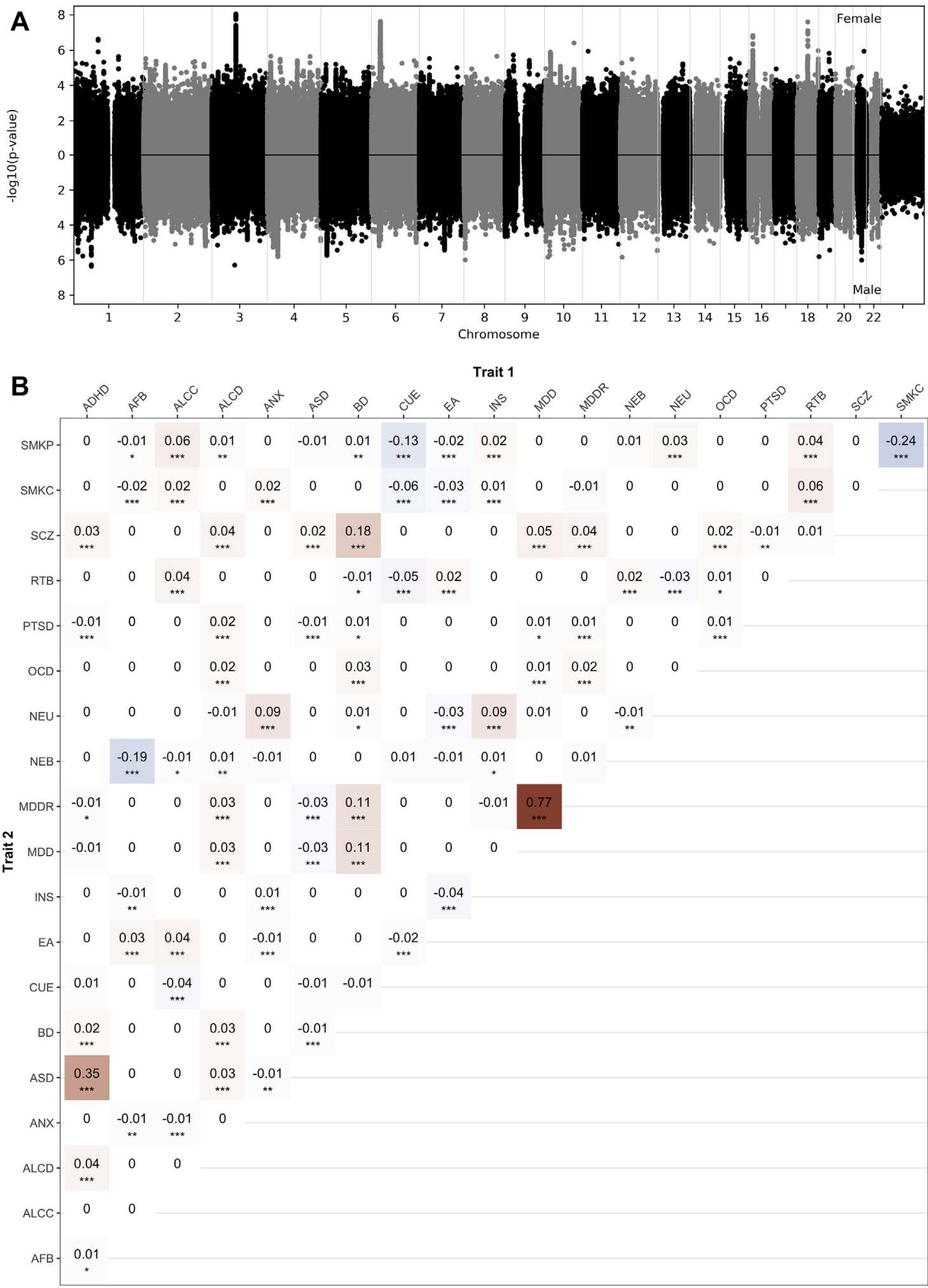

Pearson correlation coefficient

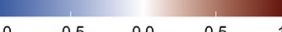


female-only (top) and male-only (bottom) lifetime cannabis use GWASs, where we observed several associations that are stronger in females (e.g., chromosomes 3, 6, 16, and 18). As cohorts for lifetime cannabis use are of very similar size, the power to detect association in both sexes is similar.

A gene-based analysis in MAGMA revealed several traits with significant sex-differentiated effects. Gene-based analysis Manhattan plots are shown in Figure S5 in Supplement 1. Traits with significant gene associations include number of children born (GLB1L2), risk-taking behavior (HFE2 and AGO2), and schizophrenia (SLTM). SLTM, which is highly expressed in cerebellum (Genotype-Tissue Expression Portal, www.gtexportal.org), was also identified in a larger (and therefore better-powered) gene-based gene-by-sex interaction for schizophrenia and across schizophrenia, bipolar disorder (BD), and MDD (20). The full set of gene-based MAGMA association statistics is provided in Table S7 in Supplement 3. None of these 4 genes showing differential sex association with the traits shows a significant differential gene expression in the brain tissues from the Genotype-Tissue Expression project v8 (Table S8 in Supplement 2).

\section{Shared Sex-Differentiated Effects Across Traits}

Many psychiatric traits are frequently comorbid and genetically correlated (13); thus, we hypothesized that sex differences in genetic effects might be a property of the SNP or gene, in which case we would expect that the sex difference observed at an SNP or gene would be observed across multiple traits. To test this hypothesis, for each pair of traits, we calculated the Pearson correlation coefficient between the SNP-based $z$ scores (i.e., scores reflecting sex-differentiated effects). Figure 2B shows a correlation matrix for pairs of traits. We observed small-to-moderate, but significant, correlations of $z$ scores for several trait pairs. The correlation of $z$ scores between MDD and recurrent MDD was high, but not equal to 1 $(r=.77, p<.001)$, indicating that there are both shared and trait-specific variants with sex-differentiated effects for these two overlapping definitions of MDD, although it should be noted that subtle differences in population structure could also impact these results. Furthermore, we observed cross-trait sharing of sex-dependent genetic effects between ASD and $\mathrm{ADHD}$ as well as BD and schizophrenia, to name examples.

\section{Gene Set Enrichment Analysis of Genes With Sex- Differentiated Effects Across Traits}

To investigate the biological function of the genes harboring SNPs with sex-differentiated genetic effects, we selected the top $0.1 \%$ of genes from each trait (Table S9 in Supplement 2), resulting in 346 genes that were mapped for gene set enrichment analysis. The top 100 gene sets enriched for genes with sex-differentiated effects are listed in Table S10 in Supplement 2. The gene sets enriched for sex-differentiated effects included neurogenesis, regulation of nervous system development, regulation of neuron differentiation, neuron differentiation, positive regulation of nervous system development, regulation of neuron projection development, and neuron development, among others.

\section{Between-Trait, Within-Sex Genetic Correlation Analysis}

The within-sex, between-trait genetic correlation results are presented as network plots (Figure $3 \mathrm{~A}-\mathrm{C}$ ) and heatmaps (Figure S6 in Supplement 1). Most between-trait genetic correlations were not significantly different between males and females (Figure 3B, C). We detected several significant sex differences in the between-trait genetic correlations; see Table 2 and Figure $3 \mathrm{~A}$ for top results and Table S11 in Supplement 2 for details. For example, educational attainment and risk-taking behavior were positively correlated in females but negatively correlated in males. Lifetime cannabis use and neuroticism were negatively correlated in females but positively correlated in males. The magnitude of $r_{g}$ was significantly greater in females than in males for a number of traits (e.g., risk-taking behavior and schizophrenia) and significantly smaller in females than in males for several trait pairs (e.g., number of children born and risk-taking behavior). Finally, we also observed trait pairs for which the estimated $r_{g}$ in one sex did not differ significantly from zero (Table S11 in Supplement 2), suggesting that either there was no significant genetic correlation between a given trait pair in one sex or the power to estimate this effect was too low.

\section{DISCUSSION}

We investigated sex differences in the genetic architecture of 20 neuropsychiatric and behavioral traits using sex-stratified autosomal GWAS summary statistics. We used 3 complementary approaches, including estimation of SNP-based heritability, genetic correlation, and heterogeneity analyses, to evaluate sex differences within traits and across trait pairs. In line with the small effect sizes of individual common variants contributing to neuropsychiatric and behavioral phenotypes (see studies referenced in Table 1), our results suggest that sex differences in the common autosomal genetic architecture of these phenotypes are also small and polygenic, indicating that larger samples will be needed to detect these differences at the individual variant level. A corollary of this conclusion is that the large sex differences in prevalence of many psychiatric conditions are not fully explained by genetic factors and are more

\footnotetext{
Figure 2. Sharing of variants with sex-differentiated effects between traits. (A) Miami plot for female-only (top) and male-only (bottom) genome-wide association studies for cannabis use (ever): female cases: $N=17,244$; male cases: $N=17,414$. For each single nucleotide polymorphism, we computed $z$ scores using Equation 1. (B) Matrix of the Pearson correlation coefficients for pairs of traits. We performed Pearson's correlation of $z$ scores and a block jackknife approach to estimate the significance of the correlation for all pairs of traits. The estimated significance of the coefficients is denoted as follows: ${ }^{\star} p<.05,{ }^{\star \star} p<$ $.01,{ }^{* \star *} p<.001$. Color coding represents positive (red) or negative (blue) correlation. ADHD, attention-deficit/hyperactivity disorder; AFB, age at first birth; ALCC, alcohol use; ALCD, alcohol dependence; ANX, anxiety disorders; ASD, autism spectrum disorder; BD, bipolar disorder; CUE, cannabis use (ever); EA, educational attainment; INS, insomnia; MDD, major depressive disorder; MDDR, major depressive disorder recurrent; NEB, number of children ever born; NEU, neuroticism; OCD, obsessive-compulsive disorder; PTSD, posttraumatic stress disorder; RTB, risk-taking behavior; SCZ, schizophrenia; SMKC, smoking (current); SMKP, smoking (previous).
} 
A
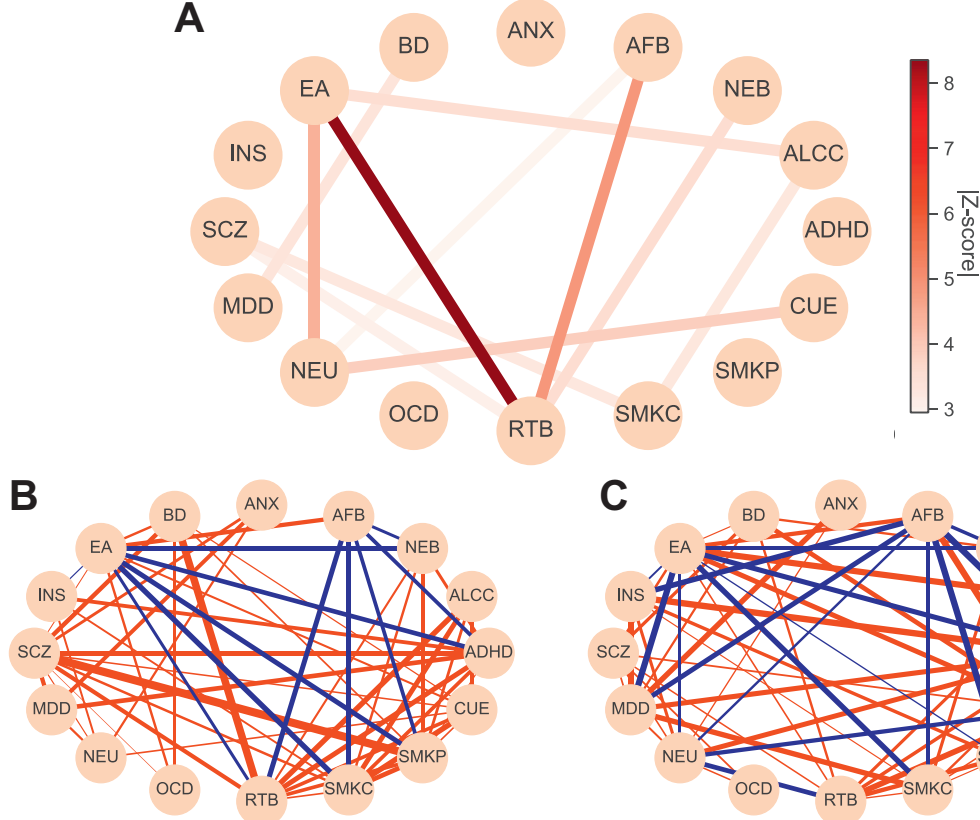

C

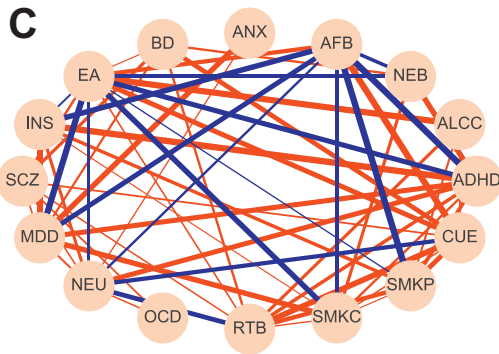

Figure 3. (A) Network plot showing between-trait genetic correlations with a significant sex difference as computed by $z$ score. The edge color represents the absolute value of the $z$ score for the difference in genetic correlation between the same 2 phenotypes in females vs. males. Only pairs of traits with false discovery rate corrected $q<.05$ sex difference are shown. (B, C) Between-trait, within-sex genetic correlation analysis. Network plots for genetic correlation estimates $\left(r_{g}\right)$ for pairs of traits in (B) males and (C) females, where each node represents a trait, and the edge represents positive (red) or negative (blue) genetic correlation. The thickness of the edge represents $-\log _{10}(q$ value) of correlation significance. Only genetic correlations with false discovery rate corrected $q<.05$ are shown. Genetic correlations were visualized using the Python package Networkx (50) and Matplotlib (51). ADHD, attentiondeficit/hyperactivity disorder; AFB, age at first birth; ALCC, alcohol use; ANX, anxiety disorders; ASD, autism spectrum disorder; BD, bipolar disorder; CUE, cannabis use (ever); EA, educational attainment; INS, insomnia; MDD, major depressive disorder; NEB, number of children ever born; NEU, neuroticism; OCD, obsessive-compulsive disorder; RTB, risk-taking behavior; SCZ, schizophrenia; SMKC, smoking (current); SMKP, smoking (previous). likely due to environmental, social, and systems-level biological differences. We caution, however, that it would be a mistake to interpret from these conclusions that genetic factors are unimportant in understanding phenotypic sex differences. As observed, even when genetic differences are small and dispersed throughout the genome, quantification of such differences can provide insight into biological processes that may impact both sexes but may be more detectable in one sex. Furthermore, the interaction between genetic risk and gendered social environments is likely to be complex, and much more research is needed to understand the effect of their interplay on mental health traits. Even with these limitations and complexities, we identified a small number of significant sex differences, described below.

For most traits and cross-trait pairs, we detected no consistent evidence of sex differences in SNP- $h^{2}$, and the genetic correlations between males and females were moderate to high (mostly $r_{g}>0.8$ ). This is consistent with prior twin-based studies that report limited evidence for substantial sex differences in heritability $(40,41)$. Equivalent heritability does not preclude the possibility of sex differences in genetic architecture. However, these findings together suggest that most common autosomal genetic effects on psychiatric phenotypes are shared across sexes.

The phenotypes that showed sex differences were among those with the largest available sample sizes, indicating that sample size impacts power to detect sex differences, and consequently, the lack of significant differences for a given phenotype may be due to limited power resulting from small sample sizes (Table S12 in Supplement 2). For example, a recent larger analysis of gene-by-sex interaction in schizophrenia, BD, and MDD revealed significant associations for schizophrenia and MDD
(20). We found that some pairs of genetically correlated traits also share sex-differentiated associations (e.g., ASD and ADHD; BD and schizophrenia). Taken together, these findings suggest that sex differences in the genetic architecture of neuropsychiatric and behavioral traits exist but are small and polygenic. They further support the hypothesis that SNPs with sex-differentiated genetic effects for one trait are also likely to exhibit sex-differentiated effects in phenotypically associated traits $(18,42)$. Moreover, we found that the set of genes with the most sex-differentiated effects across all traits is enriched (among other gene sets) for neurogenesis, neuron differentiation, and development of nervous system gene functions.

For two traits with well-powered GWAS data (educational attainment and risk-taking behavior), several interesting results emerged. Both traits demonstrated similar SNP- $h^{2}$ in males and females, indicating that there was no appreciable difference in the overall contribution of genetic factors in each sex. Also, neither trait demonstrated an excess of variants with sexdifferentiated effects, showing that (at current sample sizes) there were few detectable sex-differentiated genetic effects. However, while the genetic correlation between males and females was high [educational attainment: $r_{g}=0.92$, SE $=0.02$, as previously reported (29); risk-taking behavior: $r_{g}=0.81, \mathrm{SE}=$ 0.04], it was significantly less than 1 for both traits. These two traits were positively genetically correlated in females $\left(r_{g}=\right.$ $0.19)$ but negatively correlated in males $\left(r_{g}=-0.14\right)$. These results may be explained by a scenario in which a large number of SNPs exist with very small sex-differentiated effects, which we remain underpowered to detect at individual loci but can observe in analyses of cumulative sex differences. An alternative possibility is that there are sex differences in ascertainment and measurement [e.g., research participation rates 
Table 2. Top Results of Sex Differences in Cross-Trait Genetic Correlation Estimates

\begin{tabular}{|c|c|c|c|c|c|c|c|c|c|}
\hline \multirow[b]{2}{*}{ Trait 1} & \multirow[b]{2}{*}{ Trait 2} & \multicolumn{3}{|c|}{ Females } & \multicolumn{3}{|c|}{ Males } & \multicolumn{2}{|c|}{ Sex Difference } \\
\hline & & $r_{g}$ & SE & $q$ Value $_{R}$ & $r_{g}$ & SE & $q$ value $_{R}$ & z Score & $q$ Value \\
\hline$\overline{E A}$ & RTB & 0.187 & 0.033 & $6.38 \times 10^{-8}$ & -0.144 & 0.033 & $4.29 \times 10^{-5}$ & -8.353 & $7.98 \times 10^{-15}$ \\
\hline AFB & RTB & -0.035 & 0.046 & .52 & -0.344 & 0.054 & $1.23 \times 10^{-9}$ & -4.906 & $5.58 \times 10^{-5}$ \\
\hline EA & NEU & -0.22 & 0.029 & $1.72 \times 10^{-13}$ & -0.064 & 0.029 & .051 & 4.421 & $3.94 \times 10^{-4}$ \\
\hline CUE & NEU & -0.142 & 0.055 & .022 & 0.124 & 0.054 & .044 & 3.866 & $3.32 \times 10^{-3}$ \\
\hline NEB & RTB & 0.116 & 0.063 & .12 & 0.413 & 0.074 & $1.43 \times 10^{-7}$ & 3.582 & $8.19 \times 10^{-3}$ \\
\hline ALCC & EA & 0.276 & 0.047 & $2.52 \times 10^{-8}$ & 0.043 & 0.049 & .47 & -3.53 & $8.30 \times 10^{-3}$ \\
\hline $\mathrm{SCZ}$ & SMKC & 0.034 & 0.045 & .52 & 0.214 & 0.046 & $1.54 \times 10^{-5}$ & 3.301 & .013 \\
\hline ALCC & SMKC & 0.013 & 0.058 & .86 & 0.292 & 0.069 & $8.97 \times 10^{-5}$ & 3.326 & .013 \\
\hline $\mathrm{BD}$ & MDD & 0.565 & 0.079 & $4.95 \times 10^{-12}$ & 0.057 & 0.142 & .74 & -3.367 & .013 \\
\hline RTB & $\mathrm{SCZ}$ & 0.326 & 0.043 & $3.13 \times 10^{-13}$ & 0.157 & 0.038 & $1.07 \times 10^{-4}$ & -3.088 & .024 \\
\hline AFB & NEU & -0.173 & 0.037 & $1.44 \times 10^{-5}$ & -0.028 & 0.048 & .63 & 2.95 & .035 \\
\hline
\end{tabular}

The $z$ scores were calculated using equation 1 .

AFB, age at first birth; ALCC, alcohol use; BD, bipolar disorder; CUE, cannabis use (ever); EA, educational attainment; MDD, major depressive disorder; NEB, number of children ever born; NEU, neuroticism; RTB, risk-taking behavior; SCZ, schizophrenia; SMKC, smoking (current).

(43), or male and female subjects interpret the question about being a risk-taker differently], thus resulting in analysis of slightly different traits in males and females. Sex differences in ascertainment can impact genetic discovery; although such biases do not impact estimation of genetic correlation (43), they could theoretically impact sex differences in cross-trait genetic correlation or differences in heritability. In general, ascertainment effects (e.g., recruitment and participation biases) and measurement issues (e.g., phenotyping biases) should be carefully considered in future genetic studies of sex differences, for example, by using cohorts that are not subject to ascertainment biases (e.g., iPSYCH) or employing methods to mitigate this bias, such as inverse-probability weighted regression (43). Many of the current GWASs of behavioral traits are based on data from the UK Biobank (which is a relatively older, healthier, and wealthier female-biased cohort relative to the overall UK population) (44), whereas the case-control neuropsychiatric traits are typically ascertained from clinical populations.

These observations have important implications for the future of sex differences research. Although the majority of genetic effects for neuropsychiatric and behavioral traits are similar for males and females, sex-differentiated genetic effects can be identified, and we have shown for the first time that a portion are shared across traits. Comprehensive discovery of these effects will require larger sample sizes than for detection of main effects because of reduced statistical power in assessing the interaction between sex and genotype. We expect that as sample sizes increase, sex differences will continue to emerge but will be small in magnitude, reflecting the polygenic architecture of the phenotypes. For traits that are genetically correlated, we expect to observe cross-trait sharing of a portion of sex-differentiated genetic effects, as we have reported here. Furthermore, the large sex differences in prevalence of psychiatric disorders are unlikely to be explained entirely by common autosomal genetic factors. Additional studies investigating the interaction between cumulative genetic effects (including nonautosomal and rare variation), sex- differentiated cellular environments (e.g., the impact of sex hormones on genome regulation), and gendered social environments will be needed.

\section{Limitations and Considerations}

We focused on neuropsychiatric and behavioral traits with available sex-stratified autosomal GWAS summary statistics. The GWAS cohorts we analyzed consisted exclusively of individuals of European ancestry, and thus we are unable to assess the degree to which these results are applicable to other ancestries. It is essential that future GWASs analyze cohorts representing diverse ancestries for a more comprehensive and inclusive analysis of sex differences. Furthermore, lack of access to genotype-level data restricted our analyses to methods developed for summary statistics. This precluded testing some hypotheses, such as the possibility of sexspecific genetic liability thresholds, which is most directly tested by comparing the polygenic score distributions in male and female subjects (15). Additionally, ascertainment and participation bias may confound identification of true sex differences (43). Estimation of SNP- $h^{2}$ relies on several important assumptions (e.g., regarding the underlying genetic architecture and number of causal variants) $(29,30)$ and can be influenced by many factors (e.g., sex-specific population prevalences, sex-dependent ascertainment methods for cases and controls, different sample sizes in males and females) (45-47). Accurate estimation of sex-specific population prevalences is complex given potential sex differences in referral, with underdiagnosis in one sex [e.g., as seen for ADHD (48)]. To account for these issues, we used prevalence estimates from two different populations (Denmark and United States) and a second method (LDAK) to test for consistency of results under different assumptions. SNP- $h^{2}$ estimates based on the two different population prevalence estimates were highly correlated, indicating that in the absence of sex-specific ascertainment biases varying substantially by country, results using prevalence rates based on other populations (e.g., 
United Kingdom, from where many of the study participants are drawn) would likely be consistent as well. There were substantial differences in estimation based on either LDSC or LDAK, likely owing to the different model assumptions related to genetic architecture; the biggest discrepancies were for the traits with the smallest sample sizes (Figure S3 in Supplement 1); the true SNP- $h^{2}$ estimate is likely to fall in between these estimates. Furthermore, it is likely that some of the GWAS summary statistics may have included data from super-screened and unscreened control subjects, which may have biased upward the genetic correlation estimates (49). Clear best practices for sex-specific genetic analyses have not yet been established and are needed for future studies.

The most direct method to identify SNPs with sexdependent effects is to perform a genotype-by-sex interaction test. However, this requires individual-level genotype data. A sex-stratified analysis followed by a difference test, such as the $z$ score used here, is equivalent to a genotype-by-sex interaction test when there is no interaction between covariates (e.g., principal components, age) and the strata (e.g., male and female) and the trait variances are equivalent in the two strata (33). If those assumptions hold, our stratified analyses will be conservative. Conversely, if those assumptions are violated, our stratified analysis will be robust to those covariate interactions and differences in residual variances when evaluating whether the common variant effects are heterogeneous across sex. For example, we have previously shown that $p$ values from a genotype-by-sex interaction test were highly correlated with $z$ score $p$ values from the sex-stratified analysis (autosomal SNPs $r=.65, p<2.2 \times 10^{-16}$, X chromosome SNPs $r=.71, p<2.2 \times 10^{-16}$ ) in analysis of obsessivecompulsive disorder (22). However, subsequent systematic analysis of larger cohorts may illuminate whether these assumptions are violated and their impact on the interpretation of variants with sex-differentiated effects.

Another important limitation of our study is that we assessed only autosomal genetic effects, as summary statistics from the sex chromosomes were not available for the traits we analyzed. The sex chromosomes are frequently excluded from GWASs, owing to special consideration required for quality control and analyses, with many methods not allowing for the inclusion of sex chromosomes.

\section{Conclusions}

Through within- and between-trait analyses, we find preliminary and modest evidence of sex-dependent autosomal genetic effects, with no single SNP exhibiting significant sexdifferentiated genetic effects across neuropsychiatric and behavioral phenotypes among cohorts of European ancestry. However, consistent with the observed effect sizes of discovery GWASs of these phenotypes, these effects are small and polygenic, and therefore larger samples are needed to comprehensively identify these effects and characterize their functional contribution to complex traits. Furthermore, studies of sex differences taking into account nonautosomal and rare genetic variants as well as environmental (e.g., endogenous hormonal influences and exogenous exposures due to one's sex), ethnic, and cultural differences are needed.

\section{ACKNOWLEDGMENTS AND DISCLOSURES}

This work was supported by the Wellcome Trust (Grant No. 106047 [to JM]), Sêr Cymru II COFUND Fellowship from the Welsh Government (to JM), National Institutes of Health (Grant No. 5U01MH109539 [to RKW], Grant Nos. R01NS102371-01A1, R01MH113362, U01HG009086, R01MH118223, and RM1HG009034 [to LKD], Grant No. R01CA229618 [to BES], Grant Nos. R00MH101367 and R01MH119243 [to PHL], and Grant No. MH109532 [to HJE]), Stanley Center for Psychiatric Research (to RKW), National Institute for Health Research (NIHR) as part of the Maudsley Biomedical Research Centre (to JRIC and GB), European Union Horizon 2020 Programme for research and innovation (H2020/2014-2020) (Grant No. 667302 [CoCA] [to ADB], Grant Nos. 667302 and 728018 [to SVF], and Grant No. 847879 [PRIME] [to JB]), European Union Seventh Framework Programme for research, technological development and demonstration (Grant No. 602805 [to SVF]), National Institute of Mental Health (Grant Nos. 5R01MH101519 and U01 MH109536-01 [to SVF], Grant No. K01MH113848 [to REP], and Grant No. R01MH114924 [to LAW and MT]), Brain \& Behavior Research Foundation (Grant No. 28632 P\&S Fund [to REP]), and National Institute of Neurological Disorders and Stroke (Grant Nos. R01 NS102371 and R01 NS105746 [to CAM]).

High-performance computing facilities were funded with capital equipment grants from the Guy's and St. Thomas' Charity (Grant No. TR130505) and Maudsley Charity (Grant No. 980).

The iPSYCH team was supported by the Lundbeck Foundation (Grant Nos. R165-2013-15320, R102-A9118, R155-2014-1724, and R248-20172003) and the universities and university hospitals of Aarhus and Copenhagen. The Danish National Biobank resource was supported by the Novo Nordisk Foundation. Data handling and analysis at GenomeDK were supported by the National Institute of Mental Health (Grant No. 1U01MH109514-01 [to ADB]). High-performance computer capacity for handling and statistical analysis of iPSYCH data at GenomeDK was provided by the Center for Genomics and Personalized Medicine and the Centre for Integrative Sequencing, iSEQ, Aarhus University, Denmark (grant to $A D B)$.

This work utilized the computational resources of the Dutch national e-infrastructure with the support of SURF Cooperative (https://userinfo. surfsara.nl/).

The original GWAS data were supported by National Institute of Mental Health Psychiatric Genomics Consortium (PGC) grants (Grant Nos. U01 MH109528, U01 MH109539, U01 MH109536, U01 MH109501, U01

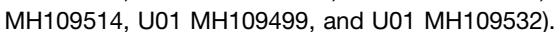

This study represents independent research partly funded by the NIHR Biomedical Research Centre at South London and Maudsley National Health Service Foundation Trust and King's College London. The views expressed are those of the authors and not necessarily those of the National Health Service, the NIHR, or the Department of Health and Social Care.

The other members of the Sex Differences Cross-Disorder Analysis Group of the PGC are Martin Alda, Marco Bortolato, Christie L. Burton, Enda Byrne, Caitlin E. Carey, Lauren Erdman, Laura M. Huckins, Manuel Mattheisen, Elise Robinson, and Eli Stahl.

$\mathrm{JM}$ and EAK were responsible for study conception, design, analyses, and writing. SBG and GAMB were responsible for analyses and writing. MT, $\mathrm{RKW}, \mathrm{CH}, \mathrm{JRIC}$, and $\mathrm{GB}$ were responsible for analyses and editing. ADB, DD, JG, TW, JB, CMB, PHL, CAM, REP, SJW, NW, HJE, WG, YY, BMN, SVF, TLP, LAW, and LED were responsible for editing. JMG and JWS were responsible for writing. BES and LKD were responsible for writing and analytic supervision.

We thank Dr. Helena Gaspar for providing a python script that was used to estimate differences in linkage disequilibrium score regression genetic correlation across sex and Donald Hucks for computing population prevalence by sex for the BioVU (Vanderbilt University Medical Center biobank) population. We also thank the following consortia and groups that contributed data: Attention Deficit Hyperactivity Disorder Working Group of the PGC and iPSYCH, Autism Spectrum Disorder Working Group of the PGC and iPSYCH, Bipolar Disorder Working Group of the PGC, Major Depressive Disorder Working Group of the PGC, Obsessive Compulsive Disorder Working Group of the PGC, Post-traumatic Stress Disorder Working Group of the PGC, Schizophrenia Working Group of the PGC, Substance Use 
Disorders Working Group of the PGC, and UK Biobank GWAS results generated by the Neale Lab (http://www.nealelab.is/uk-biobank).

CMB has received a grant from and is a Scientific Advisory Board member of Shire, is a consultant for Idorsia Pharmaceuticals, and has received author royalties from Pearson. EAK is employed by Janssen Pharmaceutical Companies of Johnson \& Johnson. All other authors report no biomedical financial interests or potential conflicts of interest.

\section{ARTICLE INFORMATION}

From the MRC Centre for Neuropsychiatric Genetics and Genomics (JM), Division of Psychological Medicine and Clinical Neurosciences, Cardiff University, Cardiff; Social, Genetic and Developmental Psychiatry Centre (CH, JRIC, GB), Institute of Psychiatry, Psychology \& Neuroscience, King's College London; National Institute for Health Research Maudsley Biomedical Research Centre (JRIC, GB), South London and Maudsley National Health Service Trust, London, United Kingdom; Section of Genetic Medicine (EAK, BES), Department of Medicine and Institute for Genomics and Systems Biology, University of Chicago; Center for Genetic Medicine (BES), Department of Pharmacology, Northwestern University, Chicago, Illinois; Computational Sciences (EAK), Janssen Pharmaceuticals, Spring House, Pennsylvania; Department of Molecular Physiology and Biophysics (SBG), Division of Genetic Medicine (LKD), Department of Medicine, and Department of Psychiatry and Behavioral Sciences (LKD), Vanderbilt University Medical Center, Nashville, Tennessee; Psychiatric \& Neurodevelopmental Genetics Unit (GAMB, JWS), Department of Psychiatry (PHL, JMG, TLP), Massachusetts General Hospital; Center for Genomic Medicine (GAMB), Massachusetts General Hospital; Analytic and Translational Genetics Unit (RKW, BMN), Department of Medicine, Massachusetts General Hospital; Department of Obstetrics and Gynecology (JMG), Massachusetts General Hospital, Harvard Medical School, Boston; Stanley Center for Psychiatric Research (RKW), Broad Institute of MIT and Harvard, Cambridge, Massachusetts; Department of Psychiatry (MT, LAW), Institute for Human Genetics (MT, LAW), and Weill Institute for Neurosciences (MT, LAW), University of California San Francisco, San Francisco; Department of Psychiatry and Behavioral Sciences (LED), Stanford University, Stanford, California; Departments of Psychiatry (CMB) and Nutrition (CMB), University of North Carolina at Chapel Hill, Chapel Hill, North Carolina; Department of Psychiatry (CAM) and Genetics Institute (CAM), University of Florida, Gainesville, Florida; Department of Psychiatry (REP) and Virginia Institute for Psychiatric and Behavioral Genetics (REP), Virginia Commonwealth University, Richmond, Virginia; Department of Health Sciences Research (SJW), Division of Biomedical Statistics and Informatics, Mayo Clinic, Rochester, Minnesota; Department of Biochemistry and Molecular Biology (HJE), Indiana University School of Medicine, Indianapolis, Indiana; Genetic Epidemiology Research Branch (WG), National Institute of Mental Health, National Institutes of Health, Bethesda, Maryland; Departments of Psychiatry (SVF) and Neuroscience and Physiology (SVF), State University of New York Upstate Medical University, Syracuse, New York; Department of Psychiatry and Neuropsychology (GAMB), School for Mental Health and Neuroscience, Faculty of Health, Medicine, and Life Sciences, Maastricht University, Maastricht; Department of Human Genetics (JB), Radboud University Medical Center, Nijmegen, The Netherlands; Department of Medical Epidemiology and Biostatistics (CH, CMB), Karolinska Institutet, Stockholm, Sweden; The Lundbeck Foundation Initiative for Integrative Psychiatric Research (ADB, DD, JG), iPSYCH, Aarhus; Department of Biomedicine (ADB, DD, JG), Aarhus University; Center for Genomics and Personalized Medicine (ADB, DD, JG), Aarhus, Denmark; Institute of Biological Psychiatry (TW), Mental Health Center Sct. Hans, Mental Health Services Copenhagen; Department of Clinical Medicine (TW), Faculty of Health, University of Copenhagen; Section for GeoGenetics (TW), GLOBE Institute, University of Copenhagen; The Lundbeck Foundation Initiative for Integrative Psychiatric Research (iPSYCH) (TW), Copenhagen, Denmark; Institute for Molecular Bioscience (NW), University of Queensland, Brisbane, Australia; and School of Life Sciences (YY), Fudan University, Shanghai, China.

$\mathrm{JM}$ and EAK contributed equally to this work.

BES and LKD jointly supervised this work.

Address correspondence to Joanna Martin, Ph.D., at martinjm1@cardiff. ac.uk, or Lea K. Davis, Ph.D., at lea.k.davis@gmail.com.

Received May 14, 2020; revised Dec 15, 2020; accepted Dec 17, 2020.
Supplementary material cited in this article is available online at https:// doi.org/10.1016/j.biopsych.2020.12.024.

\section{REFERENCES}

1. Westergaard D, Moseley P, Sørup FKH, Baldi P, Brunak S (2019): Population-wide analysis of differences in disease progression patterns in men and women. Nat Commun 10:1-14.

2. Traglia M, Bseiso D, Gusev A, Adviento B, Park DS, Mefford JA, et al. (2017): Genetic mechanisms leading to sex differences across common diseases and anthropometric traits. Genetics 205:979-992.

3. Rawlik K, Canela-Xandri O, Tenesa A (2016): Evidence for sex-specific genetic architectures across a spectrum of human complex traits. Genome Biol 17:166.

4. Khramtsova EA, Davis LK, Stranger BE (2019): The role of sex in the genomics of human complex traits. Nat Rev Genet 20:173-190.

5. Naqvi S, Godfrey AK, Hughes JF, Goodheart ML, Mitchell RN, Page DC (2019): Conservation, acquisition, and functional impact of sex-biased gene expression in mammals. Science 365:eaaw7317.

6. Eaton NR, Keyes KM, Krueger RF, Balsis S, Skodol AE, Markon KE, et al. (2012): An invariant dimensional liability model of gender differences in mental disorder prevalence: Evidence from a national sample. J Abnorm Psychol 121:282-288.

7. Plana-Ripoll O, Pedersen CB, Holtz Y, Benros ME, Dalsgaard S, de Jonge $P$, et al. (2019): Exploring comorbidity within mental disorders among a Danish national population. JAMA Psychiatry 76:259-270.

8. Rutter M, Caspi A, Moffitt TE (2003): Using sex differences in psychopathology to study causal mechanisms: Unifying issues and research strategies. J Child Psychol Psychiatry 44:1092-1115.

9. Goldstein JM, Holsen L, Huang G, Hammond BD, James-Todd T, Cherkerzian S, et al. (2016): Prenatal stress-immune programming of sex differences in comorbidity of depression and obesity/metabolic syndrome. Dialogues Clin Neurosci 18:425-436.

10. Goldstein JM, Hale T, Foster SL, Tobet SA, Handa RJ (2019): Sex differences in major depression and comorbidity of cardiometabolic disorders: Impact of prenatal stress and immune exposures. Neuropsychopharmacology 44:59-70.

11. Mareckova K, Holsen LM, Admon R, Makris N, Seidman L, Buka S, et al. (2016): Brain activity and connectivity in response to negative affective stimuli: Impact of dysphoric mood and sex across diagnoses. Hum Brain Mapp 37:3733-3744.

12. Cross-Disorder Group of the Psychiatric Genomics Consortium (2019): Genomic relationships, novel loci, and pleiotropic mechanisms across eight psychiatric disorders. Cell 179:1469-1482.e11.

13. Brainstorm Consortium, Anttila V, Bulik-Sullivan B, Finucane HK, Walters RK, Bras J, et al. (2018): Analysis of shared heritability in common disorders of the brain. Science 360:eaap8757.

14. Bulik-Sullivan BK, Finucane HK, Anttila V, Gusev A, Day FR, Loh PR, et al. (2015): An atlas of genetic correlations across human diseases and traits. Nat Genet 47:1236-1241.

15. Martin J, Walters RK, Demontis D, Mattheisen M, Lee SH, Robinson E, et al. (2018): A genetic investigation of sex bias in the prevalence of attention-deficit/hyperactivity disorder. Biol Psychiatry 83:1044-1053.

16. Walters RK, Polimanti R, Johnson EC, McClintick JN, Adams MJ, Adkins AE, et al. (2018): Transancestral GWAS of alcohol dependence reveals common genetic underpinnings with psychiatric disorders. Nat Neurosci 21:1656-1669.

17. Neale L: Rapid GWAS of thousands of phenotypes for 337,000 samples in the UK Biobank Available at: http://www.nealelab.is/ blog/2017/7/19/rapid-gwas-of-thousands-of-phenotypes-for-337000samples-in-the-uk-biobank. Accessed October 10, 2019

18. Mitra I, Tsang K, Ladd-Acosta C, Croen LA, Aldinger KA, Hendren RL, et al. (2016): Pleiotropic mechanisms indicated for sex differences in autism. PLoS Genet 12:e1006425.

19. Grove J, Ripke S, Als TD, Mattheisen M, Walters RK, Won H, et al. (2019): Identification of common genetic risk variants for autism spectrum disorder. Nat Genet 51:431-444.

20. Blokland GAM, Grove J, Chen CY, Cotsapas C, Tobet S, Handa R, et al. (2020): Sex-dependent shared and non-shared genetic 
architecture across mood and psychotic disorders. bioRxiv. https:// doi.org/10.1101/2020.08.13.249813.

21. Hammerschlag AR, Stringer S, de Leeuw CA, Sniekers S, Taskesen E, Watanabe K, et al. (2017): Genome-wide association analysis of insomnia complaints identifies risk genes and genetic overlap with psychiatric and metabolic traits. Nat Genet 49:1584-1592.

22. Khramtsova EA, Heldman R, Derks EM, Tourette Syndrome/ Obsessive-Compulsive Disorder Working Group of the Psychiatric Genomics Consortium (2019): Sex differences in the genetic architecture of obsessive-compulsive disorder. In: Davis LK, Stranger BE, editors. Am J Med Genet B Neuropsychiatr Genet 180:351-364.

23. Duncan LE, Ratanatharathorn A, Aiello AE, Almli LM, Amstadter AB, Ashley-Koch AE, et al. (2018): Largest GWAS of PTSD ( $N=20$ 070) yields genetic overlap with schizophrenia and sex differences in heritability. Mol Psychiatry 23:666-673.

24. Strawbridge RJ, Ward J, Lyall LM, Tunbridge EM, Cullen B, Graham N, et al. (2018): Genetics of self-reported risk-taking behaviour, transethnic consistency and relevance to brain gene expression. Transl Psychiatry 8:178.

25. Clarke TK, Adams MJ, Davies G, Howard DM, Hall LS, Padmanabhan S, et al. (2017): Genome-wide association study of alcohol consumption and genetic overlap with other health-related traits in UK Biobank ( $N=112$ 117). Mol Psychiatry 22:1376-1384.

26. Schumann G, Liu C, O’Reilly P, Gao H, Song P, Xu B, et al. (2016): KLB is associated with alcohol drinking, and its gene product $\beta$-Klotho is necessary for FGF21 regulation of alcohol preference. Proc Natl Acad Sci U S A 113:14372-14377.

27. Barban N, Jansen R, de Vlaming R, Vaez A, Mandemakers JJ, Tropf FC, et al. (2016): Genome-wide analysis identifies 12 loci influencing human reproductive behavior. Nat Genet 48:1462-1472.

28. Okbay A, Beauchamp JP, Fontana MA, Lee JJ, Pers TH, Rietveld CA, et al. (2016): Genome-wide association study identifies 74 loci associated with educational attainment. Nature 533:539-542.

29. Hübel C, Gaspar HA, Coleman JRI, Finucane H, Purves KL, Hanscombe KB, et al. (2019): Genomics of body fat percentage may contribute to sex bias in anorexia nervosa. Am J Med Genet B Neuropsychiatr Genet 180:428-438.

30. Bulik-Sullivan BK, Loh PR, Finucane HK, Ripke S, Yang J, Schizophrenia Working Group of the Psychiatric Genomics Consortium, et al. (2015): LD Score regression distinguishes confounding from polygenicity in genome-wide association studies. Nat Genet 47:291-295.

31. Speed D, Balding DJ (2019): SumHer better estimates the SNP heritability of complex traits from summary statistics. Nat Genet 51:277284.

32. Roden DM, Pulley JM, Basford MA, Bernard GR, Clayton EW, Balser JR, et al. (2008): Development of a large-scale de-identified DNA biobank to enable personalized medicine. Clin Pharmacol Ther 84:362-369.

33. Pedersen CB, Mors O, Bertelsen A, Waltoft BL, Agerbo E, McGrath JJ, et al. (2014): A comprehensive nationwide study of the incidence rate and lifetime risk for treated mental disorders. JAMA Psychiatry 71:573-581.

34. Winkler TW, Justice AE, Cupples LA, Kronenberg F, Kutalik Z, Heid IM, et al. (2017): Approaches to detect genetic effects that differ between two strata in genome-wide meta-analyses: Recommendations based on a systematic evaluation. PLoS One 12:e0181038.
35. Kunsch HR (1989): The jackknife and the bootstrap for general stationary observations. Ann Stat 17:1217-1241.

36. Busing FMTA, Meijer E, Van Der Leeden R (1999): Delete-m Jackknife for Unequal m. Stat Comput 9:3-8.

37. Watanabe K, Taskesen E, van Bochoven A, Posthuma D (2017): Functional mapping and annotation of genetic associations with FUMA. Nat Commun 8:1826.

38. Watanabe K, Stringer S, Frei O, Mirkov MU, Polderman TJC, van der Sluis S, et al. (2019): A global view of pleiotropy and genetic architecture in complex traits. Nat Genet 51:1339-1348.

39. Dalsgaard S, Thorsteinsson E, Trabjerg BB, Schullehner J, PlanaRipoll O, Brikell I, et al. (2020): Incidence rates and cumulative incidences of the full spectrum of diagnosed mental disorders in childhood and adolescence. JAMA Psychiatry 77:155-164.

40. Polderman TJ, Benyamin B, de Leeuw CA, Sullivan PF, van Bochoven A, Visscher PM, et al. (2015): Meta-analysis of the heritability of human traits based on fifty years of twin studies. Nat Genet 47:702-709.

41. Bernabeu E, Canela-Xandri O, Rawlik K, Talenti A, Prendergast J, Tenesa A (2020): Sexual differences in genetic architecture in UK Biobank. bioRxiv. https://doi.org/10.1101/2020.07.20.211813.

42. Winkler TW, Justice AE, Graff M, Barata L, Feitosa MF, Chu S, et al. (2015): The influence of age and sex on genetic associations with adult body size and shape: A large-scale genome-wide interaction study. PLoS Genet 11:e1005378.

43. Pirastu N, Cordioli M, Nandakumar P, Mignogna G, Abdellaoui A, Hollis B, et al. (2021): Genetic analyses identify widespread sexdifferential participation bias [published online ahead of print Apr 22]. Nat Genet.

44. Fry A, Littlejohns TJ, Sudlow C, Doherty N, Adamska L, Sprosen T, et al. (2017): Comparison of sociodemographic and health-related characteristics of UK Biobank participants with those of the general population. Am J Epidemiol 186:1026-1034.

45. van Rheenen W, Peyrot WJ, Schork AJ, Hong Lee S, Wray NR (2019): Genetic correlations of polygenic disease traits: From theory to practice. Nat Rev Genet 20:567-581.

46. Schork A, Hougaard D, Nordentoft M, Mors O, Boerglum A, Mortensen PB, et al. (2019): Exploring contributors to variability in estimates of SNP-heritability and genetic correlations from the iPSYCH case-cohort and published meta-studies of major psychiatric disorders. bioRxiv. https://doi.org/10.1101/487116.

47. Trzaskowski M, Mehta D, Peyrot WJ, Hawkes D, Davies D, Howard DM, et al. (2019): Quantifying between-cohort and betweensex genetic heterogeneity in major depressive disorder. Am J Med Genet B Neuropsychiatr Genet 180:439-447.

48. Staller J, Faraone SV (2006): Attention-deficit hyperactivity disorder in girls. CNS Drugs 20:107-123.

49. Kendler KS, Chatzinakos C, Bacanu S (2020): The impact on estimations of genetic correlations by the use of super-normal, unscreened, and family-history screened controls in genome wide case-control studies. Genet Epidemiol 44:283-289.

50. U.S. Department of Energy Office of Scientific and Technical Information: Exploring network structure, dynamics, and function using NetworkX Available at: https://www.osti.gov/biblio/960616. Accessed January 15, 2019.

51. Hunter JD (2007): Matplotlib: A 2D graphics environment. Comput Sci Eng 9:90-95. 